\title{
Intervenções psicológicas para crianças que vivem ou convivem com HIV/Aids
}

\author{
Evelise Rigoni de Faria - Universidade Federal do Rio Grande do Sul, Porto Alegre, Brasil \\ Milena da Rosa Silva - Universidade Federal do Rio Grande do Sul, Porto Alegre, Brasil \\ Helena Pillar Kessler - Universidade Federal do Rio Grande do Sul, Porto Alegre, Brasil \\ Tonantrin Ribeiro Gonçalves - Universidade Federal do Rio Grande do Sul, Porto Alegre, Brasil \\ Fernanda Torres de Carvalho - Universidade Federal do Rio Grande do Sul, Porto Alegre, Brasil \\ Cesar Augusto Piccinini - Universidade Federal do Rio Grande do Sul, Porto Alegre, Brasil
}

\begin{abstract}
Resumo
Com o avanço no tratamento antirretroviral, a infecção pelo HIV/Aids em crianças passou de um quadro agudo para uma doença crônica, tendo implicações médicas, psicológicas e sociais. Aspectos da convivência com o HIV/Aids, como revelação do diagnóstico, adesão ao tratamento e enfrentamento de diversos lutos, são especialmente desafiadores entre as crianças. Assim, este estudo buscou identificar, na literatura, intervenções psicológicas desenvolvidas nos últimos anos para crianças que vivem ou convivem com o HIV/Aids. Foram revisadas as bases de dados Medline, PsycInfo, Indexpsi, PEPSIC, SciELO e LILACS, encontrando-se 17 estudos sobre o tema. Foram descritos objetivos, público-alvo, país de origem, contextualização teórica, técnicas utilizadas e resultados dessas intervenções. A revisão apontou que são raros estudos descrevendo intervenções psicológicas para essas crianças. Além disso, muitos dos estudos encontrados não apresentaram uma clara descrição do método utilizado e avaliação de seus resultados, o que prejudica a utilização dessas intervenções em outros contextos.
\end{abstract}

Palavras-chave: HIV, AIDS, Crianças, Intervenções psicológicas.

\section{Psychological interventions to children infected and affected by HIV/Aids}

\begin{abstract}
Due to the recent progress with antiretroviral treatment, HIV/Aids infection has changed from an acute clinical status to a chronic illness with medical, psychological and social implications. Typical aspects of living with HIV/Aids, such as diagnostic disclosure, treatment adherence and the coping with several mournings, are particularly challenging among children. Therefore, this study aimed to identify psychological interventions that have been developed in the past years with children infected or affected by HIV/Aids. Medline/Pubmed, PsycInfo, Indexpsi, PEPSIC, SciELO and LILACS databases were reviewed, where 17 studies about the related subject were found. Objectives, target population, theoretical conceptualization, used techniques and results of these interventions have been described. The review indicates that studies describing psychological interventions for these children are rare. Moreover, many of these studies did not present a clear description of the methods used and the evaluation of the results, limiting the use of these interventions in other contexts.
\end{abstract}

Keywords: HIV, AIDS, Children, Psychological interventions.

\section{Intervenciones psicológicas para los niños que viven o conviven con el VIH/SIDA}

\begin{abstract}
Resumen
Con los avances en la terapia antirretroviral, la infección por VIH/SIDA en los niños cambió de enfermedad aguda a enfermedad crónica, con implicaciones médicas, psicológicas y sociales. Aspectos de la convivencia con el VIH/SIDA, como la revelación del diagnóstico, la adherencia al tratamiento y afrontamiento a las pérdidas, son especialmente difíciles para los niños. Por eso, este estudio buscó identificar en la literatura intervenciones psicológicas desarrolladas en los últimos años para niños que conviven con el VIH/SIDA. Fueron revisados el Medline, PsycInfo, Indexpsi, PEPSIC, SciELO y LILACS, en donde se encontraron 17 estudios sobre el tema. Se describen los objetivos, público objetivo, país de origen, el contexto teórico, técnicas utilizadas y los resultados de esas intervenciones. La revisión apuntó que son raros los estudios que describen las intervenciones psicológicas para esos niños. Además, muchos de los estudios encontrados no presentan claramente el método usado y la evaluación de sus resultados, lo que perjudica el uso de esas intervenciones en otros contextos.

Palabras clave: VIH, SIDA, Niños, Intervenciones psicológicas.
\end{abstract}

Desde que foi inicialmente descrita, no início dos anos oitenta, a epidemia de HIV/Aids ${ }^{1}$ vem passando por mudanças epidemiológicas significativas, dentre as

\footnotetext{
${ }^{1}$ A infecção pelo HIV inclui diversas fases de progressão até o quadro de Aids caracterizado por imunodeficiência grave. Para fins de clareza, se utilizará o termo HIV/Aids para referir tanto a situações de infecção, que ainda não apresentam sintomas da imunodeficiência, como ao quadro clínico de Aids já desenvolvido.

Disponivel em www.scielo.br
}

quais se destaca o aumento de casos de infecção através da transmissão heterossexual (Forsyth, 2003). Segundo dados do Ministério da Saúde (Brasil, 2010), no Brasil, atualmente, a principal via de transmissão do vírus entre indivíduos com 13 anos de idade ou mais se dá dessa forma, tanto para homens quanto para mulheres (47\% e $97 \%$ dos casos notificados em 2009, respectivamente). Outra mudança importante diz respeito à redução da taxa de mortalidade decorrente 
da doença devido ao avanço nas possibilidades de tratamento, de forma que a infecção pelo HIV/Aids passou a ter um caráter crônico (Brasil, 2010).

Diante dessa nova configuração da doença, as crianças vivendo com HIV/Aids passaram a compor um grupo que requer atenção especial. No Brasil, 86\% dos casos de Aids em crianças menores de 13 anos ocorre de forma vertical, ou seja, da mãe para o filho (Brasil, 2010), ainda que sejam grandes os esforços para reduzir esses números. Com os recentes avanços no tratamento antirretroviral, a expectativa de vida das crianças com HIV/Aids aumentou consideravelmente. Isso trouxe à tona uma série de questões que antes não estavam presentes, ligadas às condições sociais, físicas e psicológicas de desenvolvimento dessas crianças (Earls, Raviola, \& Carlson, 2008).

Quanto ao estudo dos aspectos psicológicos das crianças vivendo com $\mathrm{HIV} / \mathrm{Aids}$, um dos primeiros pontos a chamar a atenção dos pesquisadores foram os efeitos do vírus no sistema nervoso central de crianças e o consequente atraso no desenvolvimento cognitivo e motor (Lwin \& Melvin, 2001). Esses atrasos acometem de $8 \%$ a $13 \%$ das crianças infectadas (Forsyth, 2003). Além disso, pelo fato de a infecção por HIV ter sido, desde seu início, associada ao uso de drogas e à atividade sexual (Forsyth, 2003), juntamente com o medo, a falta de conhecimento, a vergonha e os tabus (Abadia-Barrero \& Castro, 2006), o estigma relacionado à doença ainda é muito grande. Dessa forma, os indivíduos e as famílias que vivem com este vírus sofrem severas consequências sociais e psicológicas. Isso leva muitas famílias a decidirem por manter o diagnóstico em segredo, com frequência também não revelando às crianças infectadas sua condição. Os motivos para que os pais prefiram não revelar o diagnóstico ao filho são diversos; dentre eles, estão o medo de que ele não consiga manter o sigilo e a culpa muitas vezes sentida pelos pais por terem sido responsáveis pela infecção da criança (Blasini e cols., 2004; Thomason, Bachanas, \& Campos, 1996). Lester e cols. (2002) salientaram que a demora para realizar essa revelação pode ter consequências negativas para a criança, incluindo aumento de problemas psicológicos e comportamentais, luto mais complicado e prejuízo na compreensão e adesão ao tratamento.

Outro aspecto da vida de crianças vivendo com $\mathrm{HIV} /$ Aids que tem recebido atenção dos pesquisadores é a adesão ao tratamento, que engloba tanto questões de ordem prática quanto psicológica. O tratamento antirretroviral traz dificuldades na medida em que exige rigorosa adesão, necessária por toda a vida, uma vez que interrupções no tratamento resultam em rápida progressão da doença (Simoni, Amico, Pearson, \& Malow, 2008). No caso das crianças, a adesão ao tratamento se torna ainda mais complexa devido a fatores tais como o grande número de comprimidos, seu tamanho, consistência e gosto desagradáveis, e a necessidade de levar as medicações para a escola ou para outros lugares que a criança frequenta, o que revelaria a doença a pessoas de fora da família. Além disso, as crianças dependem de um cuidador que administre o seu tratamento, tornando, assim, a dedicação do cuidador um fator crucial para a saúde das crianças (Simoni e cols., 2007).

Tanto as crianças infectadas pelo HIV/Aids como as que não possuem o vírus mas convivem com ele são filhas de pais vivendo com HIV/Aids - sofrem com todos os estressores associados a uma doença crônica. A família toda se envolve e precisa lidar com a doença, que repercute de diferentes formas em cada membro, muitas vezes obrigando a família a se reorganizar para poder enfrentar as adversidades que se apresentam (Rotheram-Borus, Flannery, Rice, \& Lester, 2005). A partir disso, é importante que se implementem intervenções voltadas para essa população de crianças que vivem ou convivem com o HIV/Aids, visando ajudá-las a enfrentar os desafios impostos pela presença da infecção.

Considerando o impacto da infecção pelo HIV/Aids sobre as crianças portadoras, a atual possibilidade de que estas tenham uma longa sobrevida e cheguem à adolescência, adultez e velhice, bem como a necessidade de assistência multiprofissional, torna-se relevante identificar intervenções psicológicas que vêm sendo desenvolvidas nos últimos anos para atender a essa demanda. Assim, o presente estudo teve por objetivo fazer uma revisão da literatura para identificar e caracterizar as intervenções psicológicas dirigidas às crianças que vivem ou convivem com o HIV/Aids.

\section{Método}

Foi realizada uma seleção de artigos científicos envolvendo intervenções psicológicas para crianças que vivem ou convivem com o HIV/Aids, publicados no período de janeiro de 1980 a maio de 2010 . A estratégia de busca incluiu uma combinação de diversos termos que contemplavam infância, infecção pelo HIV/Aids e intervenções psicológicas. Por exemplo, para infância utilizaram-se termos como child, infant e pediatric, e para intervenções, termos como psychotherapy, counseling, intervention e health education. Quando possível, foram adicionados termos convencionados e a mesma estratégia foi utilizada, com pequenas modificações, nas diferentes bases de dados. As bases de dados revisadas foram Medline (Pubmed), PsycInfo, Indexpsi, PEPSIC, SciELO e LILACS. 
Entre todos os 1.537 resumos obtidos nas buscas, foram selecionados 55 estudos que se referiam explicitamente ao tema investigado, os quais foram lidos na íntegra. Destes, foram incluídos na revisão aqueles que preenchiam os seguintes critérios: ter como população-alvo das intervenções crianças que vivem ou convivem com o HIV/Aids; apresentar enfoque psicológico; ser escrito em inglês, português ou espanhol; e oferecer algum detalhamento da intervenção proposta. A partir desses critérios, foram selecionados 17 estudos.

Realizou-se uma análise descritiva das intervenções, buscando identificar suas características gerais. Também foi realizada uma classificação das mesmas com base em seus referenciais teóricos, destacando-se três principais: 1) psicanalítico; 2) familiar-sistêmico; e 3) cognitivo-comportamental. Ainda houve alguns estudos que não definiram seu referencial teórico e que configuraram um quarto grupo. Inicialmente, as intervenções são descritas de maneira conjunta, e, posteriormente, apresenta-se a caracterização dessas intervenções.

\section{Resultados e Discussão}

Após a leitura dos artigos na íntegra, buscou-se caracterizá-los para apresentar um panorama geral das intervenções. Os dados levantados estão relacionados na Tabela 1.

Tabela 1. Caracterização geral das intervenções

\begin{tabular}{|c|c|c|}
\hline & $\%$ & $\mathbf{N}$ \\
\hline \multicolumn{3}{|l|}{ Ano publicação } \\
\hline 1990-1999 & 35,3 & 6 \\
\hline $2000-2010$ & 64,7 & 11 \\
\hline \multicolumn{3}{|l|}{ País de origem } \\
\hline EUA & 64,7 & 11 \\
\hline Reino Unido & 5,9 & 1 \\
\hline Canadá & 5,9 & 1 \\
\hline Polônia & 5,9 & 1 \\
\hline Uganda & 5,9 & 1 \\
\hline Porto Rico & 5,9 & 1 \\
\hline Brasil & 5,9 & 1 \\
\hline \multicolumn{3}{|l|}{ Público-alvo } \\
\hline Crianças que vivem com HIV & 58,9 & 10 \\
\hline Crianças que convivem com HIV & 17,6 & 3 \\
\hline Crianças que vivem e que convivem com HIV & 23,5 & 4 \\
\hline \multicolumn{3}{|l|}{ Modalidade $^{2}$} \\
\hline Familiar & 64,7 & 11 \\
\hline Grupal & 47,1 & 8 \\
\hline Individual & 41,1 & 7 \\
\hline \multicolumn{3}{|l|}{ Avaliação de resultados } \\
\hline Sim & 64,7 & 11 \\
\hline Não & 35,3 & 6 \\
\hline \multicolumn{3}{|l|}{ Objetivo } \\
\hline Desenvolver ou melhorar capacidades psicológicas & 64,7 & 11 \\
\hline Aumentar adesão ao tratamento & 11,7 & 2 \\
\hline Auxiliar a família na revelação do diagnóstico & 11,7 & 2 \\
\hline Desenvolver estratégias de controle da dor em procedimentos médicos & 5,9 & 1 \\
\hline Planejar cuidados da criança após a morte dos pais & 5,9 & 1 \\
\hline \multicolumn{3}{|l|}{ Referencial teórico 3} \\
\hline Psicanalítico & 35,3 & 6 \\
\hline Familiar-sistêmico & 29,4 & 5 \\
\hline Cognitivo-comportamental & 11,7 & 2 \\
\hline Não definido & 29,4 & 5 \\
\hline
\end{tabular}

\footnotetext{
${ }^{2}$ Algumas intervenções utilizaram mais de uma das modalidades descritas e, por isso, a soma dos percentuais é superior a 100.

${ }^{3}$ Algumas intervenções basearam-se em mais de um referencial teórico e, por isso, a soma dos percentuais é superior a 100.

Psico-USF, Bragança Paulista, v. 18, n. 1, p. 65-76, jan./ abril 2013
} 
Como pode ser observado, o desenvolvimento de intervenções psicológicas para crianças que vivem ou convivem com o HIV/Aids vem apresentando um crescimento ao longo dos anos, embora o número de publicações a esse respeito ainda seja pequeno, principalmente se considerarmos o elevado número de intervenções que vêm sendo realizadas com adultos nesse contexto (Faria, Carvalho, Gonçalves, Moskovics, \& Piccinini, 2011). Entre os estudos revisados, os Estados Unidos lideraram no número de produções, tendo sido desenvolvidas $64,7 \%$ dessas intervenções. Os demais países - Reino Unido, Canadá, Polônia, Uganda, Porto Rico e Brasil - tiveram uma intervenção cada um, representando $5,9 \%$ do total.

Em relação ao público-alvo das intervenções, a maioria destas focalizava crianças com HIV/Aids, sendo 10 intervenções $(58,9 \%)$ voltadas especificamente a essas crianças e quatro $(23,5 \%)$ para crianças que vivem e que convivem com HIV/Aids. Ainda foram encontradas três intervenções $(17,6 \%)$ que focalizavam especificamente crianças que convivem com HIV/Aids. Quanto à modalidade da intervenção, foram identificadas intervenções realizadas tanto em formato familiar quanto grupal e/ou individual, prevalecendo as primeiras. Observou-se, contudo, que muitas intervenções combinavam encontros com a família com encontros individuais ou grupais. Quanto à avaliação das intervenções realizadas, a maioria dos artigos $(64,7 \%)$ referiu alguma forma de avaliação dos resultados. Algumas dessas avaliações, contudo, não apresentavam qualquer sistematização.

No que se refere aos objetivos, observa-se que a maioria das intervenções revisadas $(64,7 \%)$ tinha por objetivo desenvolver capacidades psicológicas da criança que podem ser afetadas diante do contexto do HIV/Aids, como é o caso de habilidades sociais, estratégias de enfrentamento e autoconceito. A revelação do diagnóstico e a adesão ao tratamento também foram o foco de algumas intervenções $(11,7 \%$ cada), enquanto um número menor delas deteve-se sobre situações específicas de controle da dor diante de procedimentos médicos $(5,9 \%)$ e planejamento dos cuidados da criança após morte dos pais $(5,9 \%)$.

Conforme critério de inclusão da presente revisão, para serem consideradas intervenções psicológicas, estas deveriam ter um enfoque psicológico e ser embasadas em algum referencial teórico da área da psicologia. Após caracterização geral das intervenções identificadas, estas foram classificadas considerando a teoria que embasava a técnica utilizada. Cabe ressaltar que algumas das intervenções basearam-se em mais de um referencial teórico, tendo sido utilizados: o psicanalítico (6 estudos); o familiar-sistêmico (5); e o cognitivo-comportamental (2). Cinco estudos não explicitaram o referencial teórico utilizado. A seguir, apresentam-se as intervenções identificadas em cada uma dessas categorias.

\section{Intervenções com referencial psicanalítico}

Foram encontrados seis estudos descrevendo intervenções realizadas com referencial teórico psicanalítico (Aguirre \& Arruda, 2006; Gewirtz \& Gossart-Walker, 2000; Gossart-Walker \& Moss, 2000; Lovrin, 1995; Trad, Kentros, Solomon, \& Greenblatt, 1994; Willemsen \& Anscombe, 2001). Alguns desses estudos basearam-se especificamente nas teorizações de Bion a respeito do funcionamento dos grupos (Gossart-Walker \& Moss, 2000; Willemsen \& Anscombe, 2001). Outra intervenção teve como base a Psicologia do Ego (Lovrin, 1995), enquanto os demais não referiram a vertente psicanalítica utilizada.

O estudo de Gerwirtz e Gossart-Walker (2000) apresentou uma intervenção que objetivava auxiliar crianças americanas que viviam com HIV/Aids e seus familiares a alcançar maior estabilidade emocional, além de desenvolver estratégias adequadas para lidar com a doença. Os autores realizaram sessões individuais, familiares e grupais acompanhadas de orientações para os pais e apoio em questões práticas, como a garantia de direitos da criança. A intervenção ocorria na casa das famílias ou em locais de fácil acesso na comunidade. $O$ modelo considerava as especificidades de cada caso e, portanto, não havia um número de sessões ou arranjo familiar ou individual determinados previamente. Os autores não apresentaram uma avaliação formal dos resultados, porém sugeriram que esta era uma intervenção útil também para crianças que convivem com familiares portadores de HIV/Aids.

Outro estudo de um dos autores referidos (Gossart-Walker \& Moss, 2000) descreveu uma intervenção em grupo para crianças e adolescentes americanos que conviviam com familiares portadores do HIV/Aids, tendo como base a teoria de Bion e também a Teoria Sistêmica. Tinha como objetivo geral promover suporte interpessoal e auxiliar o enfrentamento da doença, mas objetivos específicos também foram delineados considerando as necessidades de cada participante. O grupo, de caráter aberto e com número ilimitado de sessões, era realizado em um serviço comunitário e cada encontro era composto por um momento de discussão e outro de brincadeira, sendo que o coordenador realizava interpretações dos comportamentos e falas expressos no grupo. Novamente, não foram identificadas evidências empíricas sobre os resultados dessa intervenção. 
Também foram localizadas outras duas intervenções grupais de base psicanalítica (Lovrin, 1995; Willemsen \& Anscombe, 2001). Lovrin (1995) propôs uma intervenção baseada na Psicologia do Ego para meninas americanas de oito anos de idade que tinham perdido seus pais ou irmãos em virtude do HIV/Aids. O estudo utilizava uma técnica denominada biblioterapia, que consistia em um grupo terapêutico que usava diferentes histórias como ferramentas para propiciar a discussão das crianças a respeito de suas experiências de vida. Enfatizava-se a resolução de problemas, buscando promover a modelagem de papéis pela identificação com os heróis da literatura, bem como o relacionamento entre pares. Os encontros foram realizados na escola durante dois semestres. Com base em análise qualitativa das sessões de um grupo, o autor destacou que a intervenção propiciou que as crianças entrassem em contato com seus sentimentos e, mediante do desenvolvimento de empatia e do apoio mútuo, diminuiu o sentimento de isolamento.

Já no estudo de Willemsen e Anscombe (2001) realizaram-se grupos de arteterapia e ludoterapia para crianças inglesas que viviam e que conviviam com HIV/Aids. As autoras destacaram a função do coordenador do grupo de conter as ansiedades das crianças, partindo das ideias de Bion. O grupo consistia de oito encontros semanais durante os quais as crianças brincavam livremente utilizando materiais lúdicos e artísticos. O terapeuta tinha postura não-diretiva, intervindo quando oportuno. Tomando por base a análise qualitativa, as autoras referiram que a intervenção propiciou maior socialização, diminuição de ansiedades relativas ao tratamento, bem como diminuição de dificuldades comportamentais e alimentares das crianças.

Foram também encontrados dois estudos de caso descrevendo tratamentos psicanalíticos conduzidos com crianças que vivem com HIV/Aids (Aguirre \& Arruda, 2006; Trad e cols., 1994). Trad e cols. (1994) descrevem uma intervenção realizada com uma criança americana de quatro anos de idade que vivia com HIV/Aids e sua mãe, também portadora. Essa intervenção tinha como objetivo ajudar a criança a reconhecer, aceitar e lidar com a própria infecção e de seus familiares, procurando ainda fortalecer a aliança familiar. Após a conclusão da intervenção, a família foi monitorada com encontros a cada duas semanas. Com base no caso apresentado, os autores defenderam que a psicoterapia promoveu a tranquilidade emocional da criança e uma intensificação dos laços familiares, fortalecendo o apoio mútuo entre mãe e filha. A partir da análise desse caso, os autores delinearam um protocolo de psicoterapia breve psicodinâmica para crianças pré-escolares vivendo com $\mathrm{HIV} /$ Aids que incluía três etapas: 1) aliança terapêutica; 2) abertura do conflito, que compreenderia o cerne do processo terapêutico; e 3) reconciliação familiar, cujo objetivo seria abrir canais de comunicação e de apoio dentro da familia.

O estudo de Aguirre e Arruda (2006) foi o único encontrado pela presente revisão que apresentou uma intervenção para crianças que vivem com HIV/Aids realizada no Brasil. Trata-se de um estudo de caso de um menino de nove anos que vivia com HIV/Aids, atendido semanalmente em psicoterapia lúdica de orientação psicodinâmica durante um ano. Os atendimentos foram realizados em serviço de psicologia da rede pública e o artigo descreveu o processo da psicoterapia com ênfase nas fantasias e defesas da criança e na relação paciente-terapeuta. De acordo com os autores, a psicoterapia propiciou benefícios ao menino, pois, após um ano de acompanhamento, ele parecia dispor de melhores recursos internos para lidar com seus conflitos, expressando com mais facilidade suas emoções.

Analisando-se conjuntamente os estudos com referencial psicanalítico, pode-se constatar que todos descreviam intervenções que procuravam auxiliar a criança no enfrentamento da doença, sua ou dos pais. Coerentemente com a orientação teórica, esse objetivo foi buscado por meio do trabalho com as fantasias e conflitos das crianças em relação ao HIV/Aids. Dois estudos (Gossart-Walker \& Moss, 2000; Lovrin, 1995) tentavam ainda promover suporte interpessoal, como uma tentativa de sobrepujar o grande isolamento físico e psicológico no qual se colocam as famílias e crianças que vivem ou convivem com o HIV/Aids. Além disso, as técnicas psicanalíticas utilizadas foram bastante variadas e, em alguns casos, eram flexíveis de acordo com as necessidades das crianças.

\section{Intervençôes com referencial familiar-sistêmico}

Foram encontrados cinco estudos descrevendo intervenções com referencial teórico familiar-sistêmico (Cunningham, Naar-King, Ellis, Pejuan, \& Secord, 2006; Ellis, Naar-King, Cunningham, \& Secord, 2006; Gossart-Walker \& Moss, 2000; Kmita e cols., 2002; O'Hare e cols., 2005). Uma delas (Gossart-Walker \& Moss, 2000) já foi apresentada na seção anterior, uma vez que se baseava predominantemente no referencial psicanalítico, embora incluísse também a abordagem familiar-sistêmica.

O estudo de Kmita, Baranska e Niemiec (2002) teve como objetivo descrever e avaliar a utilização conjunta de diferentes estratégias psicossociais voltadas à promoção do empoderamento de crianças que vivem ou convivem com HIV/Aids, além de seus familiares. 
Mais especificamente, os objetivos dessas intervenções seriam: diminuir o isolamento social das famílias, desenvolver estratégias para lidar com a doença e promover a autoestima e a autoeficácia. Para tanto, compreendiam entrevistas terapêuticas com os familiares, sessões com as crianças e com a família, grupos com as crianças e com os familiares, baseadas na psicoeducação. Essas diferentes práticas eram realizadas conforme a necessidade de cada família. Trinta crianças polonesas participaram de intervenções em dois contextos distintos: médico (hospital ou clínica de atendimento) e não-médico (acampamento terapêutico). Com base em avaliação qualitativa preliminar das intervenções, os autores afirmaram que estas propiciaram melhora no funcionamento psicológico das crianças e seus familiares, o desenvolvimento de estratégias de enfrentamento e a ampliação da rede social das famílias. Os achados apontaram ainda que as modalidades de intervenção que envolveram pais e filhos mostraram-se mais efetivas do que aquelas que trabalhavam apenas com as crianças ou com os familiares, em ambos os contextos (médico e não-médico).

A promoção de empoderamento dos cuidadores também foi o objetivo principal da intervenção descrita por O’Hare e cols. (2005). Os autores descreveram um programa de acompanhamento de crianças que viviam com HIV/Aids que haviam perdido o pai ou a mãe como consequência da Aids. Esse programa fornecia cuidados físicos, psicológicos e sociais às crianças e seus cuidadores, mediante visitas domiciliares e psicoeducação. A equipe de psicologia buscava desenvolver o empoderamento dos cuidadores por meio da educação para a saúde, especialmente com informações sobre o HIV, formas de infecção e tratamento. Também se buscava fornecer apoio para as famílias e auxílio para lidarem com as experiências de perda e luto. Análises baseadas nos dados de 172 crianças ugandenses que já haviam participado do programa mostraram um aumento na sua autoconfiança e na dos cuidadores, sendo que estes referiram terem sido beneficiados pelo programa.

As intervenções propostas por Cunningham e cols. (2006) e Ellis e cols. (2006) fazem parte de um mesmo projeto, o qual utilizou como abordagem psicoterápica a Terapia Multissistêmica (MST). Trata-se de uma estratégia intensiva de tratamento baseado em evidências, realizada em domicílio, para crianças e adolescentes com problemas clínicos severos e seus familiares. De acordo com Cunningham e cols. (2006), essa abordagem já tinha se mostrado efetiva no acompanhamento de outras condições pediátricas crônicas, como o diabetes. Seu embasamento teórico encontra-se na socioecologia e na abordagem familiar sistêmica, e constitui-se em uma psicoterapia familiar que utiliza técnicas cognitivas, comportamentais e sistêmicas, envolvendo sessões individuais e familiares.

O estudo realizado por Ellis e cols. (2006) descreveu um programa que utiliza a MST, envolvendo 21 crianças e adolescentes americanos vivendo com HIV/Aids, que teve como objetivo aumentar a adesão ao tratamento antirretroviral. Após uma avaliação inicial de cada sistema (individual, familiar, comunitário) para identificar quais fatores contribuíam para os problemas de adesão de cada criança, os terapeutas estabeleciam metas para o tratamento e as intervenções familiares. Os resultados do programa foram avaliados pelos níveis de carga viral e de questionários respondidos pelos cuidadores sobre a adesão e a infecção das crianças. Conforme os autores, não houve mudança significativa na adesão ao tratamento relatada pelos cuidadores. Contudo, houve uma diminuição significativa da carga viral em 94\% das crianças após a intervenção.

Também utilizando a MST, Cunningham e cols.(2006) relataram um estudo de caso com um menino americano de 12 anos de idade e baixa adesão ao tratamento. A duração do tratamento e o número de sessões não foram mencionados, mas os autores enfatizaram que a MST é uma abordagem flexível, cuja duração e aspectos trabalhados com cada paciente dependem de avaliação individualizada. Os autores destacaram que, após a psicoterapia, houve importante redução na carga viral do paciente. Embora não tenha sido possível determinar se esta redução se deu por maior adesão ao tratamento ou outros fatores, os autores enfatizaram que acreditavam que a psicoterapia estava associada ao resultado, tendo em vista a história do paciente e a evolução de sua condição emocional e clínica.

Analisando-se conjuntamente os estudos envolvendo o referencial familiar-sistêmico, pode-se constatar que três deles (Gossart-Walker \& Moss, 2000; Kmita e cols., 2002; O'Hare e cols., 2005) descreviam intervenções visando promover apoio interpessoal e auxiliar no enfrentamento da doença. Os dois outros estudos (Cunningham e cols., 2006; Ellis e cols., 2006) descreviam intervenções que focavam a adesão da criança aos antirretrovirais. As técnicas utilizadas foram grupais ou familiares, incluindo estratégias de psicoeducação e psicoterapia familiar.

\section{Intervenções com referencial cognitivo-comportamental}

Foram encontrados dois artigos descrevendo intervenções realizadas com referencial teórico cognitivo-comportamental (Draimin, Gamble, Shire, \& Hudis, 1998; Schiff, Holtz, Peterson, \& Rakusan, 2001). Um dos estudos (Draimin e cols., 1998) 
descreve um modelo de intervenção com o objetivo de planejar os cuidados da criança após a morte dos pais. Esse modelo, chamado de Project Care, tinha como público-alvo mães americanas vivendo com HIV/Aids e seus filhos de 8 a 11 anos. No projeto, especialistas em família e HIV/Aids visitavam as familias, ajudandoas a criar planos de vida para os filhos menores após a morte dos pais, incluindo-se planos de custódia legalmente formalizados. Os especialistas guiavam-se por módulos baseados em técnicas psicoeducativas e realizavam conversas com as mães e os filhos sobre os seguintes temas: revelação da doença e comunicação familiar; custódia e planejamento; cuidador substituto. Os autores não descreveram uma avaliação sistemática dos resultados da intervenção, mas destacaram a atenção personalizada às mães e filhos como ponto positivo. Como limitação, apontou-se a falta de apoio entre pares, já que cada família era atendida individualmente.

Schiff, Holtz, Peterson e Rakusan (2001) descreveram uma intervenção psicológica voltada para a promoção de estratégias de diminuição e controle da dor em procedimentos médicos realizados com crianças que vivem com HIV/Aids, mediante de técnicas de controle da ansiedade. Participaram do estudo 43 crianças americanas com idades entre 4 e 12 anos, acompanhadas por um cuidador, geralmente a mãe. A intervenção consistia em quatro encontros realizados em intervalos de três meses. Logo antes da criança realizar um procedimento doloroso (por exemplo, exames, coletas de sangue), um psicólogo atuava como "treinador" (coach) da dupla, orientando o cuidador a aplicar uma pomada anestésica, e a criança a realizar exercícios de respiração para o controle da ansiedade. Além disso, sugeria-se que a criança escolhesse bonecos, brinquedos ou atividades para se distrair durante o procedimento, e depois recebia uma recompensa, como figurinhas ou pequenos brinquedos. Desse modo, a intervenção incluía provimento de informação, modelagem e reforço. Os resultados indicaram diminuição significativa dos níveis de ansiedade e da percepção subjetiva de dor da criança durante procedimentos médicos invasivos.

Destaca-se que ambas as intervenções apresentavam um foco bastante específico no que concerne aos seus objetivos, o que parece coerente com o referencial teórico utilizado e favorece a avaliação de resultados, embora apenas uma delas (Schiff e cols., 2001) o tenha feito de maneira sistemática. Cabe ainda ressaltar que o objetivo da primeira intervenção (Draimin e cols., 1998), planejamento da custódia após a morte dos pais, parece também refletir uma preocupação mais intensa na época em que o estudo foi publicado, quando a terapia antirretroviral ainda era incipiente e o reconhecimento da infecção como doença crônica ainda estava se estabelecendo.

\section{Intervenções sem referencial teórico definido}

Foram encontrados cinco estudos que não explicitaram o referencial teórico utilizado (Anderson e cols., 1990; Blasini e cols., 2004; Miller \& Bortner, 1996; Salter-Goldie e cols., 2007; Wiener e cols., 1993), embora os seus objetivos e técnicas utilizadas situem essas intervenções também no campo da psicologia. O estudo realizado por Anderson e cols. (1990) apresentou um programa de intervenção para crianças americanas menores de cinco anos vivendo com HIV/Aids e seus cuidadores. O objetivo era produzir melhora nas habilidades sociais, brincadeiras, autocuidado, autoconceito, desenvolvimento cognitivoperceptual e motor das crianças. A intervenção tinha caráter interdisciplinar e as sessões incluíam educação, terapia ocupacional, terapia física, psicoterapia, fonoaudiologia, assistência social e cuidados de enfermagem, conforme as necessidades de cada criança. Além disso, a intervenção envolvia brincadeiras, jogos, exercícios físicos e desenvolvimento de adaptadores para dificuldades posturais e de motricidade, por exemplo. As crianças e seus cuidadores participavam da intervenção três vezes por semana, no serviço de saúde onde a criança realizava o seu tratamento. Os cuidadores ainda participavam de um grupo de apoio semanal. Os autores não descreveram detalhadamente como se davam esses atendimentos e também não mencionaram qualquer avaliação dos seus resultados.

Objetivos semelhantes foram buscados pela intervenção apresentada por Wiener e cols. (1993). Esta envolvia crianças americanas vivendo com HIV/Aids e seus irmãos, pais e demais familiares, portadores ou não do vírus, com o objetivo de promover satisfação pessoal, autoconfiança e estabelecimento de relações gratificantes. A intervenção consistia de grupos de apoio por teleconferência que eram realizados separadamente para cada público específico (crianças que vivem com HIV/Aids; irmãos; mães e pais portadores e não-portadores de HIV; mães adotivas; avós e avôs cuidadores). Os grupos eram realizados três vezes por semana, durante quatro a seis semanas, sendo os temas discutidos definidos pelos participantes. A avaliação da intervenção, baseada nos dados de 40 participantes, crianças e familiares, revelou avaliações mais positivas de si mesmo, diminuição do sentimento de solidão e maior habilidade dos cuidadores para lidar com a criança logo após a intervenção e no seguimento de seis meses. Os autores ainda pontuaram outras vantagens da intervenção, 
como a proteção dos participantes do contato frente a frente, o que muitos evitam por causa do preconceito, além de sobrepujar as distâncias geográficas. Os coordenadores, contudo, precisavam ser bastante habilidosos, pois contavam apenas com as comunicações verbais dos participantes durante a teleconferência.

O estudo de Miller e Bortner (1996) também descreveu um modelo de intervenção para crianças que vivem com HIV/Aids e seus cuidadores com o objetivo de desenvolver autoconhecimento e autocuidado nas crianças, além de promover a recreação e estimular a brincadeira. A intervenção consistiu em um acampamento de final de semana para os participantes com o intuito de aliviar a sobrecarga dos cuidadores de crianças americanas com doença crônica. As crianças e seus cuidadores recebiam informações sobre o tratamento e acompanhamento psicológico, conforme a necessidade. Embora os autores defendessem a importância desse tipo de intervenção, não apresentaram resultados objetivos de sua aplicação, referindo apenas que, em razão do relato dos familiares e da alta procura pelo acampamento, esta era uma experiência gratificante para as crianças e as famílias.

O estudo de Blasini e cols. (2004) incluiu 40 crianças e adolescentes porto-riquenses que viviam com HIV/Aids, com idades entre 9 e 15 anos, e seus familiares. Tinha como metas auxiliar a família na revelação do diagnóstico de $\mathrm{HIV} /$ Aids à criança e fortalecer a capacidade da família de lidar com esse diagnóstico mediante informações, apoio, possibilidade de escolha e incentivo ao respeito mútuo entre os membros da família. A intervenção foi desenvolvida por uma equipe composta por psicólogos, enfermeiros, assistentes sociais e pediatras, constituindo-se de cinco etapas: 1) treinamento dos profissionais em uma forma de revelação do diagnóstico apropriada ao nível de desenvolvimento do paciente; 2) preparação dos pais, por meio de encontros com o grupo de pares e de sessões educacionais com a equipe; 3) sessões individuais com os pacientes no decorrer do processo de revelação; 4) sessão de revelação, com o paciente, familiares e profissional de saúde; e, por fim, 5) participação em grupo de apoio durante duas a quatro semanas após a sessão de revelação e, nos meses seguintes, conforme a necessidade (Blasini e cols., 2004). A avaliação da intervenção indicou que a revelação do diagnóstico por meio do modelo proposto aumentou a adesão das crianças e adolescentes ao tratamento, trazendo também melhoras para seu ajustamento psicossocial. Nessa direção, sentimentos negativos foram menos presentes seis meses após a revelação, predominando um senso de "normalidade" e apoio familiar. Além disso, os pacientes avaliaram a revelação como um evento positivo.

Por fim, o estudo de Salter-Goldie e cols. (2007) também apresenta um protocolo de revelação do diagnóstico de HIV/Aids para crianças desenvolvido por uma equipe canadense, formada por psicólogos, psiquiatras, assistentes sociais e pediatras. Tal protocolo tinha como objetivo realizar a revelação do diagnóstico à criança que vive com HIV/Aids de modo a atender às suas necessidades específicas, bem como às de seus familiares. $O$ protocolo compreendia três momentos: 1) preparação pré-revelação - comunicação pré-revelação com a criança e preparação dos pais; 2) revelação - evento de revelação e suporte imediato; 3) pós-revelação - acompanhamento, apoio e informação. A intervenção utilizava técnicas psicoeducativas e de apoio. Não foi descrita uma aplicação específica dessa intervenção, nem uma avaliação formal dos seus resultados. Os autores, contudo, afirmaram que a revelação do diagnóstico por este protocolo poderia ajudar as famílias a alcançar um equilíbrio entre suas preocupações a respeito da criança, suas próprias necessidades de privacidade e respeito, bem como o direito das crianças de acesso à informação sobre sua saúde.

Analisando-se conjuntamente os estudos reunidos nessa categoria, que não explicitaram seu referencial teórico, pode-se constatar que três deles (Anderson e cols., 1990; Miller \& Bortner, 1996; Wiener e cols., 1993) envolveram intervenções que buscavam desenvolver capacidades psicológicas das crianças, tais como habilidades sociais, autocuidado e autoconceito. Os dois outros estudos (Blasini e cols., 2004; SalterGoldie e cols., 2007) propuseram estratégias de revelação do diagnóstico de HIV à criança. As técnicas utilizadas foram as mais variadas, incluindo abordagens grupais ou familiares, utilizando teleconferências, brincadeiras e acampamentos, além de atendimentos interdisciplinares.

\section{Considerações finais}

A revisão da literatura apresentada revela o pequeno número de estudos publicados em revistas indexadas relatando intervenções psicológicas realizadas com crianças que vivem ou convivem com HIV/Aids. Isso não condiz com a complexidade da vivência infantil e familiar nesse contexto e a necessidade de se atentar às diversas necessidades dessas crianças, incluindo as médicas, sociais e psicológicas (Earls e cols., 2008). Mais ainda, dentre os 17 estudos examinados, seis não apresentaram uma avaliação sistemática dos resultados da intervenção. 
Dentre os 11 que apresentaram avaliação dos resultados, houve variação na maneira como isso foi feito, sendo que, em alguns casos, a avaliação foi pouco objetiva. Esses achados corroboram dados anteriores, baseados em outras revisões sistemáticas da literatura, acerca da escassez de informações sobre a efetividade dessas intervenções para crianças que vivem ou convivem com HIV/Aids, que sejam embasadas em avaliações sistemáticas e com rigor metodológico (King, De Silva, Stein, \& Patel, 2009). Assim, na presente revisão, embora todos os estudos indicassem consequências positivas das intervenções para as crianças e suas famílias, não se tem clareza quanto à validade das avaliações realizadas.

Outro aspecto que chama atenção é a predominância de estudos em países desenvolvidos. A maioria dos estudos se refere a intervenções realizadas nos Estados Unidos, com raras exceções realizadas no Reino Unido, Polônia, Porto Rico, Uganda, Canadá e Brasil. Aqui, cabe destacar a questão da língua, pois na presente revisão excluíram-se artigos que não foram publicados em português, inglês ou espanhol, o que pode ter excluído estudos escritos em outras línguas. Assim, é importante destacar que esta revisão descreve, basicamente, intervenções realizadas no contexto norte-americano.

Quanto aos objetivos das intervenções, eles apontaram para algumas especificidades do trabalho com crianças que vivem ou convivem com HIV/Aids, em relação aos adultos. Por exemplo, o planejamento dos seus cuidados após a morte dos pais é uma preocupação específica com o público infantil. Já a adesão ao tratamento, a preocupação com os procedimentos dolorosos e a revelação do diagnóstico são questões que também dizem respeito aos adultos vivendo com $\mathrm{HIV} / \mathrm{Aids}$, mas que apresentam nuances e dificuldades específicas para as crianças (Seidl, Rossi, Viana, Meneses, \& Meireles, 2005). Cabe ressaltar que a literatura indica de forma consistente as dificuldades associadas à adesão em crianças (Simoni e cols., 2007), bem como à revelação do diagnóstico de HIV para essa população (Blasini e cols., 2004; Thomason e cols., 1996). Mesmo diante dessa realidade bastante complexa, poucas intervenções foram identificadas para essas finalidades específicas, o que não faz jus à seriedade da situação.

Ainda em relação aos objetivos, embora alguns temas importantes sejam pouco contemplados nas intervenções, pode-se perceber que o foco das intervenções para crianças tem sido ampliado, acompanhando as mudanças que vêm acontecendo no perfil da epidemia de HIV/Aids. Seis intervenções foram desenvolvidas nos anos 90 , quando o tratamento antirretroviral ainda não estava fortemente estabelecido, enquanto as demais ocorreram em um período mais recente, onde a infecção pelo HIV já é considerada uma doença crônica, com muitos desafios médicos, psicológicos e sociais (Earls e cols., 2008). Dessa forma, ao lado do luto pela perda dos pais e pelo temor de sua morte, mais presentes diante de quadros agudos da infecção, temas mais específicos ganharam espaço nas intervenções, como o autocuidado, a autoestima e o enfrentamento da infecção, assim como o fortalecimento das relações familiares. A ênfase nas capacidades psicológicas das crianças deve-se também ao fato de estas estarem ainda em desenvolvimento, sendo que a presença do HIV/Aids nesse momento pode causar repercussões negativas tanto em termos emocionais como cognitivos e sociais (Franklin e cols., 2005; Lwin \& Melvin, 2001).

Em relação ao embasamento teórico das intervenções, destaca-se que ele não foi apresentado em cinco artigos, o que pode estar associado ao caráter multiprofissional presente em muitas dessas intervenções, aspecto característico do campo da saúde. A diversidade de características referidas nas intervenções, que envolveram grupos, atendimentos individuais e familiares, teleconferência, entre outros, mostra a amplitude e complexidade da situação e dos objetivos a serem alcançados, o que é esperado quando se trata particularmente do desenvolvimento infantil associado ao contexto da infecção pelo HIV (Earls e cols., 2008; Rotheram-Borus e cols., 2005).

Embora tenham sido localizadas importantes iniciativas no que diz respeito ao atendimento psicológico de crianças que vivem e convivem com o HIV/Aids, a literatura mostra que estas ainda são escassas e que muitos dos estudos encontrados sobre este tema não apresentam uma clara descrição do método utilizado, nem explicitam critérios para avaliação dos resultados encontrados, o que prejudica a utilização dessas intervenções em outros contextos. Além disso, contribui com restrições para a produção de um corpo de conhecimento mais sistemático no que tange a eficiência e/ou eficácia de intervenções para crianças convivendo com HIV/Aids.

Assim, o presente estudo evidenciou a necessidade do desenvolvimento de novas intervenções psicológicas para crianças que vivem ou convivem com o HIV, bem como uma melhor caracterização dessas intervenções e da avaliação de seus resultados. É possível que outras intervenções psicológicas estejam sendo realizadas com esse público, inclusive no Brasil, todavia, não há ainda uma publicação consistente dessas informações, o que se torna um obstáculo ao acesso das mesmas para que possam ser implementadas e avaliadas pelos demais profissionais que trabalham com essas crianças. 
74 Faria, E. R. \& cols. Intervenções para crianças que vivem ou convivem com HIV/Aids

\section{Referências}

Abadia-Barrero, C. E. \& Castro, A. (2006). Experiences of stigma and access to HAART in children and adolescents living with HIV/AIDS in Brazil. Social Science and Medicine, 62(5), 1219-1228.

Aguirre, S. B. \& Arruda, S. L. S. (2006). Psicoterapia lúdica de uma criança com AIDS. Estudos de Psicologia (Natal), 23(3), 229-237.

Anderson, J., Hinojosa, J., Bedell, G., \& Kaplan, M. T. (1990). Occupational therapy for children with perinatal HIV infection. American Journal of Occupational Therapy, 44(3), 249-255.

Blasini, I., Chantry, C., Cruz, C., Ortiz, L., Salabarria, I., Scalley, N., Matos, B., Febo, I., \& Diaz, C. (2004). Disclosure model for pediatric patients living with HIV in Puerto Rico: design, implementation, and evaluation. Journal of Developmental and Behavioral Pediatrics, 25(3), 181-189.

Brasil, Ministério da Saúde (2010) Boletim Epidemiológico Aids-DST - $n^{\circ}$ VI, $27^{a}$ a $52^{a}$ semanas epidemiológicas, julho a dezembro de 2008 e $01^{a}$ a $26^{a}$ semanas epidemiológicas, janeiro a junho de 2009. Brasília: Ministério da Saúde, Secretaria de Vigilância em Saúde, Programa Nacional de DST e Aids.

Cunningham, P. B., Naar-King, S., Ellis, D. A., Pejuan, S., \& Secord, E. (2006). Achieving adherence to antiretroviral medications for pediatric HIV disease using an empirically supported treatment: a case report. Journal of Developmental and Behavioral Pediatrics, 27(1), 44-50.

Draimin, B. H., Gamble, I., Shire, A., \& Hudis, J. (1998). Improving permanency planning in families with HIV disease. Child Welfare, 77(2), 180-194.

Earls, F., Raviola, G. J., \& Carlson, M. (2008). Promoting child and adolescent mental health in the context of the HIV/AIDS pandemic with a focus on sub-Saharan Africa. Journal of Child Psychology and Psychiatry and Allied Disciplines, 49(3), 295-312.

Ellis, D. A., Naar-King, S., Cunningham, P. B., \& Secord, E. (2006). Use of multisystemic therapy to improve antiretroviral adherence and health outcomes in HIV-infected pediatric patients: evaluation of a pilot program. Aids Patient Care and STDS, 20(2), 112-121.

Faria, E. R., Carvalho, F. T., Gonçalves, T. R., Moskovics, J. M., \& Piccinini, C. A. (2011). Intervenções psicológicas para pessoas vivendo com HIV/Aids: modelos, resultados e lacunas. Revista Interamericana de Psicologia, 45(3), 339-350.

Forsyth, B. W. (2003). Psychological aspects of HIV infection in children. Child and Adolescent Psychiatric Clinics of North America, 12(3), 423-437.

Franklin, S., Lim, H. J., Rennie, K. M., Eastwood, D., Cuene, B., \& Havens, P. L. (2005). Longitudinal intellectual assessment of children with HIV infection. Journal of Clinical Psychology in Medical Settings, 12(4), 367-376.

Gewirtz, A. \& Gossart-Walker, S. (2000). Home-based treatment for children and families affected by HIV and AIDS. Dealing with stigma, secrecy, disclosure, and loss. Child and Adolescent Psychiatric Clinics of North America, 9(2), 313-330.

Gossart-Walker, S. \& Moss, N. E. (2000). An effective strategy for intervention with children and adolescents affected by HIV and AIDS. Child and Adolescent Psychiatric Clinics of North America, 9(2), 331-345.

King, E., De Silva, M., Stein, A., \& Patel, V. (2009). Interventions for improving the psychosocial wellbeing of children affected by HIV and AIDS. Cochrane Database of Systematic Reviews(2), CD006733.

Kmita, G., Baranska, M., \& Niemiec, T. (2002). Psychosocial intervention in the process of empowering families with children living with HIV/AIDS-a descriptive study. AIDS Care, 14(2), 279-284.

Lester, P., Chesney, M., Cooke, M., Weiss, R., Whalley, P., Perez, B., Glidden, D., Petru, A., Dorenbaum, A., \& Wara, D. (2002). When the time comes to talk about HIV: factors associated with diagnostic disclosure and emotional distress in HIV-infected children. Journal of Acquired Immune Deficiency Syndromes, 31(3), 309-317.

Lovrin, M. (1995). Interpersonal support among 8year-old girls who have lost their parents or siblings to AIDS. Archives of Psychiatric Nursing, 9(2), 92-98.

Lwin, R. \& Melvin, D. (2001). Paediatric HIV infection. Journal of Child Psychology and Psychiatry and Allied Disciplines, 42(4), 427-438.

Miller, S. L. \& Bortner, M. (1996). A week in the country: a model respite program. AIDS Education and Prevention, 8(2), 176-186.

O’Hare, B. A., Venables, J., Nalubeg, J. F., Nakakeeto, M., Kibirige, M., \& Southall, D.P. (2005). Home- 
based care for orphaned children infected with HIV/AIDS in Uganda. AIDS Care, 17(4), 443450 .

Rotheram-Borus, M. J., Flannery, D., Rice, E., \& Lester, P. (2005). Families living with HIV. AIDS Care, 17(8), 978-987.

Salter-Goldie, R., King, S. M., Smith, M. L., Bitnun, A., Brophy, J., Fernandes-Penney, A., Lefebvre, A., Louch, D., Macdougall, G., Moore, W., \& Read, S.E. (2007). Disclosing HIV diagnosis to infected children: a health care team's approach. Vulnerable Children and Youth Studies, 2(1), 12-16.

Schiff, W. B., Holtz, K. D., Peterson, N., \& Rakusan, T. (2001). Effect of an intervention to reduce procedural pain and distress for children with HIV infection. Journal of Pediatric Psychology, 26(7), 417427.

Seidl, E. M. F., Rossi, W. D. S., Viana, K. F., Meneses, A. K. F. D., \& Meireles, E. (2005). Crianças e adolescentes vivendo com HIV/Aids e suas famílias: aspectos psicossociais e enfrentamento. Psicologia: Teoria e Pesquisa, 21(3), 279-288.

Simoni, J. M., Amico, K. R., Pearson, C. R., \& Malow, R. (2008). Strategies for promoting adherence to antiretroviral therapy: a review of the literature. Current Infectious Disease Report, 10(6), 515-521.

Simoni, J.M., Montgomery, A., Martin, E., New, M., Demas, P. A., \& Rana, S. (2007). Adherence to antiretroviral therapy for pediatric HIV infection: a qualitative systematic review with recommendations for research and clinical management. Pediatrics, 119(6), 1371-1383.

Thomason, B. T., Bachanas, P. J., \& Campos, P. E. (1996). Cognitive behavioral interventions with persons affected by HIV/Aids. Cognitive and Behavioral Practice, 3, 417-442.

Trad, P. V., Kentros, M., Solomon, G. E., \& Greenblatt, E. R. (1994). Assessment and psychotherapeutic intervention for an HIVinfected preschool child. Journal of the American Academy of Child and Adolescent Psychiatry, 33(9), 1338-1345.

Wiener, L. S., Spencer, E. D., Davidson, R., \& Fair, C. (1993). National telephone support groups: a new avenue toward psychosocial support for hivinfected children and their families. Social Work With Groups, 16(3), 55-71.

Willemsen, H. \& Anscombe, E. (2001). Art and play group therapy for pre-school children infected and affected by HIV/AIDS. Clinical Child Psychology and Psychiatric, 6(3), 339-350.

Recebido em 11/01/2012

Reformulado em 26/02/2013

Aprovado em 05/03/2013 
Sobre os autores:

Evelise Rigoni de Faria é psicóloga (PUCRS), doutora em Psicologia (UFRGS), especialista em Psicologia Hospitalar (UFRGS) e em Saúde Mental (Grupo Hospitalar Conceição). Atualmente, é psicóloga clínica do Grupo Hospitalar Conceição, pesquisadora do NUDIF (UFRGS) e professora do Curso de Graduação em Psicologia das Faculdades Integradas de Taquara.

Milena da Rosa Silva é psicóloga (UFRGS), doutora em Psicologia (UFRGS), professora do Departamento de Psicanálise e Psicopatologia da UFRGS e pesquisadora do Núcleo de Infância e Família (NUDIF/UFRGS) e do Núcleo de Estudo, Pesquisa e Extensão em Infância e Adolescência (NEPEIA/UFRGS).

Helena Pillar Kessler é psicóloga (UFRGS) com experiência em pesquisa e extensão universitária na área de Psicanálise, com ênfase nas temáticas da infância e vulnerabilidade social.

Tonantzin Ribeiro Gonçalves é Psicóloga (UFRGS), com doutorado e pós-doutorado em Psicologia (UFRGS); professora do Programa de Pós-Graduação em Saúde Coletiva da Universidade do Vale do Rio dos Sinos e pesquisadora colaboradora do Núcleo de Infância e Família (NUDIF/UFRGS) e do Programa Primeira Infância Melhor (Secretaria de Saúde do Estado do RS).

Fernanda Torres de Carvalho é psicóloga (PUCRS), doutora em Psicologia (UFRGS), especialista em Saúde Coletiva (Escola de Saúde Pública do Rio Grande do Sul). Atualmente, é aconselhadora do Centro de Testagem e Aconselhamento do Ambulatório de Dermatologia Sanitária da Secretaria de Saúde do Estado do RS e professora do Curso de Especialização em Saúde Comunitária da UFRGS.

Cesar Augusto Piccinini é doutor e pós-doutor pela University College London (Inglaterra), é diretor do Instituto de Psicologia da Universidade Federal do Rio Grande do Sul, pesquisador do CNPq e professor do Curso de PósGraduação em Psicologia da UFRGS.

Contato com os autores:

Instituto de Psicologia - UFRGS

Rua Ramiro Barcelos, 2.600/111 - CEP 90035-006 - Porto Alegre/RS, Brasil.

E-mail: everigoni@gmail.com 